\title{
Wavefront Parameters Recovering by Using Point Spread Function
}

\author{
Olga Kalinkina ${ }^{[0000-0002-2522-8496]}$, Tatyana Ivanova ${ }^{[0000-0001-8564-243 X]}$ \\ and Julia Kushtyseva ${ }^{[0000-0003-1101-6641]}$ \\ ITMO University, 197101 Kronverksky pr. 49, bldg. A, Saint-Petersburg, Russia \\ oskalinkinaditmo.ru, tvivanovaditmo.ru, \\ julia.kushtyseva@gmail.com
}

\begin{abstract}
At various stages of the life cycle of optical systems, one of the most important tasks is quality of optical system elements assembly and alignment control. The different wavefront reconstruction algorithms have already proven themselves to be excellent assistants in this. Every year increasing technical capacities opens access to the new algorithms and the possibilities of their application. The paper considers an iterative algorithm for recovering the wavefront parameters. The parameters of the wavefront are the Zernike polynomials coefficients. The method involves using a previously known point spread function to recover Zernike polynomials coefficients. This work is devoted to the research of the defocusing influence on the convergence of the algorithm. The method is designed to control the manufacturing quality of optical systems by point image. A substantial part of the optical systems can use this method without additional equipment. It can help automate the controlled optical system adjustment process.
\end{abstract}

Keywords: Point Spread Function, Wavefront, Zernike Polynomials, Optimization, Aberrations.

\section{Introduction}

At the stage of manufacturing optical systems, one of the most important tasks is quality of optical system elements assembly and alignment control. There are various methods for solving this problem, for example, interference methods. However, in some cases, one of which is the alignment of the telescope during its operation, other control methods are required $[1,2]$, for example control by point image (point spread function) or the image of another known object. Among the advantages of using this method for telescopic systems control is economic expediency. Firstly, there is no need for external intervention in a working telescope. Secondly, equipment that allows recording the point spread function has the lowest cost in comparison with interferometric control methods equipment.

Copyright (C) 2020 for this paper by its authors. Use permitted under Creative Commons License Attribution 4.0 International (CC BY 4.0). 
Wavefront reconstruction algorithms have been widely developed in holography, adaptive optics, microscopy, and other fields [3]. Some algorithms restore the distribution of the wavefront in the form of a sample of values, others allow you to restore the describing parameters. A necessary condition for the operation of such algorithms is the assumption that in the process of wavefront propagation through the optical system, only the phase component changes, while the amplitude component remains constant.

The Gerchberg - Saxton algorithm, and its modifications are the most frequently used and found in the literature. It is an iterative reconstruction of the phase information from the known amplitudes of the sampled image and diffraction plane intensity pictures measured. By given the amplitude of a signal and its Fourier transform, the algorithm attempts to recover the phase information for the Fourier transform, and thereby reconstruct the signal.

The algorithm alternates between Fourier and inverse Fourier transforms, using the input amplitudes at each iteration to improve the phase estimates. Using this idea, many algorithms were developed with the next inputs data - the two or more related images that differ in a certain phase diversity. It is noted that using more images increases the speed and convergence of the algorithms.

In this paper, we solve the problem of reconstructing the wavefront from only one known point image. The purpose of this work is to develop an algorithm for determining the wavefront parameters, which are the Zernike polynomials coefficients of the wave function expansion, by known point spread function.

\section{Method}

\subsection{Zernike polynomials}

One of the most common ways to describe the wavefront profile is a Zernike polynomials fitting coefficients (1):

$$
W(\rho, \varphi)=\sum_{n} \sum_{m} C_{n m} Z_{n}^{m}(\rho, \varphi)
$$

where $\mathrm{n}, \mathrm{m}$ are the indices of polynomials with $-\mathrm{n} \leq \mathrm{m} \leq \mathrm{n}, \mathrm{n}+\mathrm{m}$ is even; $C_{n m}$ is the fitting coefficient; $Z_{n}{ }^{m}(\rho, \varphi)$ is the Zernike polynomial with the corresponding indices $\mathrm{n}, \mathrm{m} ; \varphi$ is the azimuthal angle; $\rho$ is the radial distance $(1 \geq \rho \geq 0)$.

Each Zernike fitting coefficient is representing different types of optical system aberrations. Table 1 shows the correspondence of Zernike polynomials to different types of 3rd order aberrations. These Zernike coefficients are linearly independent, thus individual aberration contributions to an overall wavefront may be isolated and quantified separately.

It should also be noted that another advantage of the Zernike polynomials is the stability to computational errors in the process of modeling optical systems on a computer or in the mathematical processing of measurement results. 
Table 1. Zernike polynomials

\begin{tabular}{|l|l|}
\hline Zernike polynomials & Aberrations \\
\hline$Z_{2}^{0}(\rho, \varphi)=2 \rho^{2}-1$ & Defocus \\
$Z_{4}^{0}(\rho, \varphi)=6 \rho^{4}-6 \rho^{2}+1$ & Spherical \\
$Z_{1}^{-1}(\rho, \varphi)=\rho \sin \varphi$ & Y Tilt \\
$Z_{1}^{1}(\rho, \varphi)=\rho \cos \varphi$ & X Tilt \\
$Z_{2}^{-2}(\rho, \varphi)=\rho^{2} \sin 2 \varphi$ & $45^{\circ}$ Astigmatism \\
$Z_{2}^{2}(\rho, \varphi)=\rho^{2} \cos 2 \varphi$ & $0^{\circ}$ Astigmatism \\
$Z_{3}^{-1}(\rho, \varphi)=\left(3 \rho^{2}-2\right) \sin 3 \varphi$ & Y Coma \\
$Z_{3}^{1}(\rho, \varphi)=\left(3 \rho^{2}-2\right) \cos 3 \varphi$ & X Coma \\
\hline
\end{tabular}

\subsection{Point spread function calculation}

The point spread function (PSF) is a two-dimensional function describe an image of point source. PSF can be calculating by inverse Fourier transform of the pupil function (2):

$$
h\left(\eta^{\prime}{ }_{x}, \eta_{y}^{\prime}\right)=\left[F^{-1}\left(f_{0}\left(\rho_{x}, \rho_{y}\right)\right)\right]^{2}
$$

where $f_{0}\left(\rho_{x}, \rho_{y}\right)$ is the pupil function, $\rho_{x}, \rho_{y}$ are the canonical pupil coordinates, $\eta^{\prime}{ }_{x}, \eta_{y}^{\prime}$ are the image canonical coordinates.

The pupil function can be described as (3):

$$
f_{0}\left(\rho_{x}, \rho_{y}\right)=\left\{\begin{array}{rr}
\tau^{1 / 2}\left(\rho_{x}, \rho_{y}\right) \cdot e^{2 \pi i W\left(\rho_{x}, \rho_{y}\right)}, & \left(\rho_{x}{ }^{2}+\rho_{y}{ }^{2}\right) \leq 1 \\
0, & \left(\rho_{x}{ }^{2}+\rho_{y}{ }^{2}\right) \geq 1
\end{array}\right.
$$

where $\tau\left(\rho_{x}, \rho_{y}\right)$ - is the pupil transmission function, $W\left(\rho_{x}, \rho_{y}\right)$ is the wave aberration function, the expression $\left(\rho_{x}{ }^{2}+\rho_{y}{ }^{2}\right) \leq 1$ defines the region inside the circle pupil.

The aberration-free PSF of optical systems of diffraction-limited quality is represented by a diffraction scattering circle, which is called an Airy disk (Figure 1a), with a central maximum at a point corresponding to an ideal image. Aberrations of the optical system and alignment errors have a noticeable effect on the image of a point object. Thus, in real optical systems, the form of PSF differs from the aberration-free one and have more complex structure. In Figure 1b, one can see the PSF recorded during the control of a real telescopic system. 


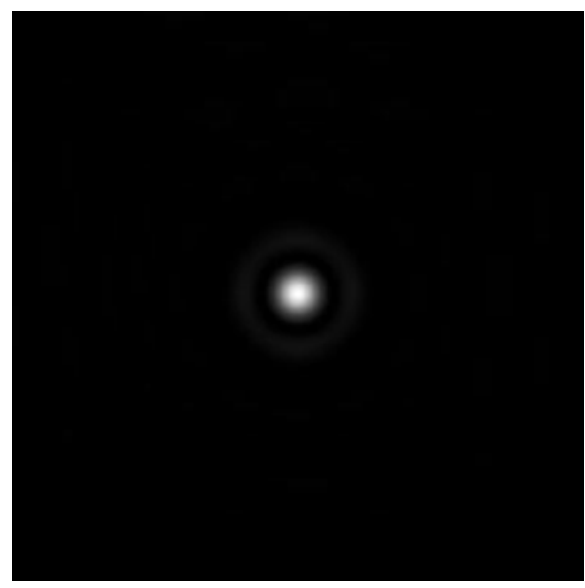

Fig. 1a. Aberration-free PSF

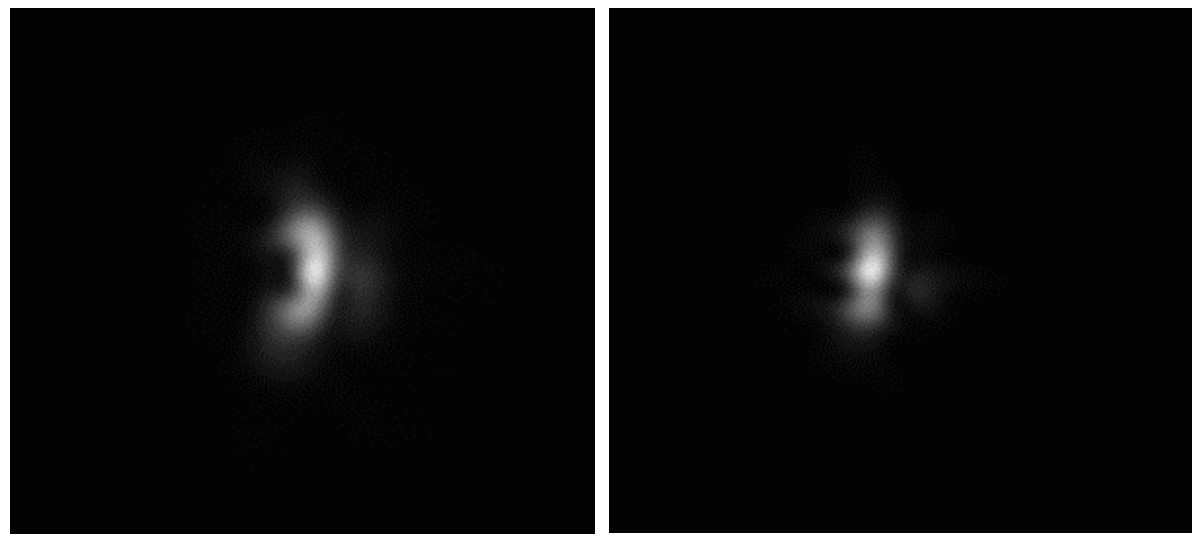

Fig.1b.PSF in real telescopic system

\subsection{Algorithm}

The direct problem in modeling optical systems is to calculate the PSF from a known set of aberrations represented by the corresponding Zernike polynomials.

The inverse problem is to restore the wavefront in the form of Zernike polynomials fitting coefficients from the known PSF intensity distribution. The inverse problem can be solved using parametric optimization. The parameters are the coefficients for Zernike polynomials. The minimized function is the standard deviation of the reference PSF from the PSF calculated at each optimization step (4) (Figure 2).

$$
\frac{1}{2} \sum_{x \prime} \sum_{y^{\prime}}\left\|h_{0 x^{\prime} y^{\prime}}-h_{x y^{\prime}}\right\|^{2} \rightarrow \min
$$




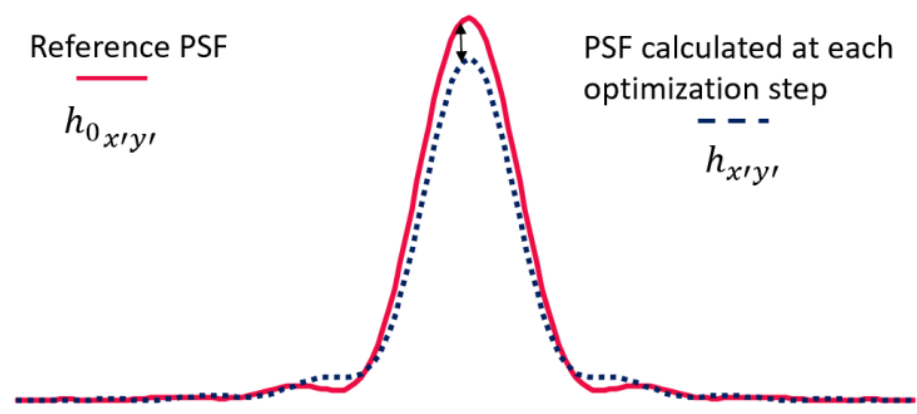

Fig.2.Minimized function

In this work, we use only four polynomials to describe wave front: astigmatism (c22 and s22 coefficients), and coma (c31 and s31 coefficients).

Modeling and solving the optimization problem is carried out using the Ceres Solver [5] library for C++ with open source code developed by Google.

Solving the optimization problem using Ceres Solver is (5):

$$
\frac{1}{2} \sum_{i}\left\|f_{i}\left(x_{i_{1}}, x_{i_{2}} \ldots x_{i_{k}}\right)\right\|^{2} \rightarrow \min _{x},-\infty<x_{j}<+\infty
$$

where $f_{i}\left(x_{i_{1}}, x_{i_{2}} \ldots x_{i_{k}}\right)$ is the objective function, $x_{i_{1}}, x_{i_{2}} \ldots x_{i_{k}}$ are optimization parameters.

As method efficiency test, we set aberration coefficients (c22, s22, c31, s31), then calculate PSF. This PSF became input for optimization and obtained during optimization coefficients can be compare with initial ones. If result coefficients differ from initial ones less, then $10^{-5}$ - we can say method work correctly.

\section{Defocusing influence on the method convergence}

The algorithm application to focused PSF, produce in some cases incorrect determination of the astigmatism coefficients (c22, s22) signs, so quite small number of PSFs with various combinations of the initial coefficients were successfully restored.

Avoiding this problem is using PSF with defocus, describe by Zernike polynomial coefficient c20. In the result sign of astigmatism is defining correctly and number of successfully restored combination of various aberration coefficients is increased.

Numerical experiments to define best defocusing value (c20 coefficient value) for stable algorithm work and successful restore aberration coefficients were performed. Thus, when checking algorithm application to a large number of modeled PSFs, the optimal value of the defocus was determined as range from 0.1 to 0.2 wavelength (absolute value). In this case aberration coefficients were successfully restored for aberration coefficients less than 0.5 wavelength (absolute value). 
6 O. Kalinkina, T. Ivanova and J. Kushtyseva

Table 2. Optimization result

\begin{tabular}{|c|c|c|c|c|}
\hline $\mathbf{c 2 0}=\mathbf{0 , 1} \boldsymbol{\lambda}$ & $\begin{array}{c}\text { initial } \\
\text { values }\end{array}$ & $\begin{array}{c}\text { optimization } \\
\text { result }\end{array}$ & $\begin{array}{c}\text { initial } \\
\text { values }\end{array}$ & $\begin{array}{c}\text { optimization } \\
\text { result }\end{array}$ \\
\hline $\mathbf{c 3 1}[\lambda]$ & 0,05689 & 0,05689 & 0,31352 & 0,31352 \\
\hline $\mathbf{s 3 1}[\lambda]$ & 0,07813 & 0,07813 & 0,35279 & 0,35279 \\
\hline $\mathbf{c 2 2}[\lambda]$ & 0,09347 & 0,09347 & 0,32016 & 0,32016 \\
\hline $\mathbf{s 2 2}[\lambda]$ & 0,06019 & 0,06019 & 0,39148 & 0,39148 \\
\hline $\begin{array}{c}\text { Number of } \\
\text { iterations }\end{array}$ & \multicolumn{2}{|c|}{5} & \multicolumn{2}{|c|}{14} \\
\hline
\end{tabular}

PSF with aberration more than 0.5 wavelength have a more complex mathematical form, which in some cases impossible to restore by optimization. But it was finding out that for every combination of coefficients c22, s22, c31, s31 is possible to find appropriate defocusing value for successful definition, and this value could be different for different coefficients combination. Figure 3 shows the PSF examples corresponding to one set of coefficients $(\mathrm{c} 22=0.2 \lambda, \mathrm{s} 22=0.6 \lambda, \mathrm{c} 31=0.6 \lambda, \mathrm{s} 31=0.4 \lambda)$ with different defocus values c20. The successful restoration were only in the one value $\mathrm{c} 20=0.6 \lambda$ (highlighted in the figure).

$c_{20}=-0,3 \lambda$

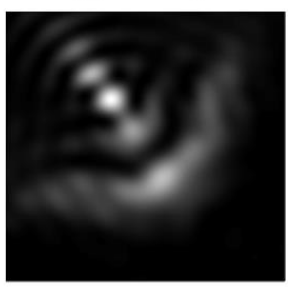

$c_{20}=0,1 \lambda$

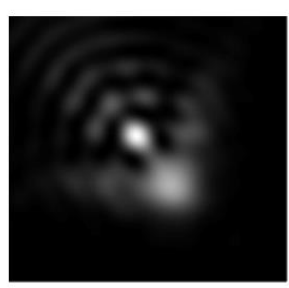

$c_{20}=-0,1 \lambda$

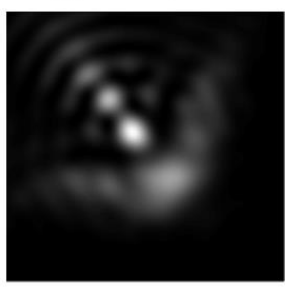

$c_{20}=0,3 \lambda$

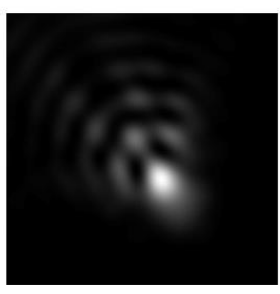

$c_{20}=0 \lambda$
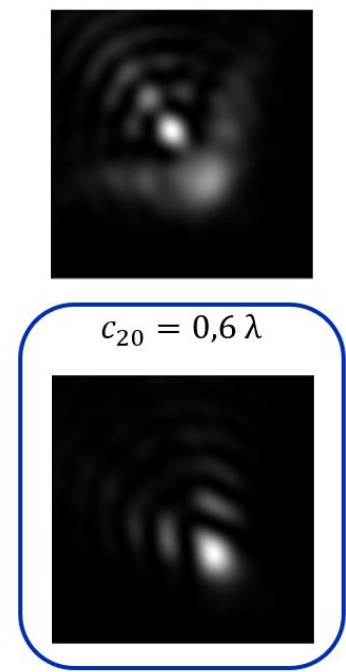

Fig.3. Example of PSF with manually selected defocus 


\section{Conclusion}

The algorithm presented in the work is intended to determine the wavefront parameters from the point spread function. Focused PSF does not provide enough information to estimate aberrations, and the presence of defocusing simplifies the determination of aberration coefficients. To determine small aberrations (less than 0.5 wavelength absolute value), the optimal defocus is in the range of 0.1-0.2 wavelength absolute value.

PSF with aberration more than 0.5 wavelength is more difficult to restore, but for every combination of coefficients $\mathrm{c} 22, \mathrm{~s} 22, \mathrm{c} 31, \mathrm{~s} 31$ is possible to find appropriate defocusing value for successful definition. However, this defocus value must be searching for each case separately.

\section{References}

1. Klebanov Y.M., Karsakov A.V., Khonina S.N., Davydov A.N., Polyakov K.A.: Compensation of wavefront aberrations in telescopes of spacecraft with adjustment of the temperature field of the telescope. Computer Optics, Volume 41, No. 1 (2017).

2. Krist J. E., Burrows C. J. Phase-retrieval analysis of pre- and post-repair Hubble Space Telescope images Applied optics - 1995, Vol. 34, No. 22 (1995).

3. Brady G.R. Application of phase retrieval to the measurement of optical surfaces and wavefronts: Submitted in partial fulfillment of the requirements for the degree Doctor of Philosophy. - University of Rochester, Rochester New York., (2008).

4. Gerchberg R.W., Saxton W.O. A practical algorithm for the determination of phase from image and diffraction plane pictures OPTIC, Vol. 35, No. 2 (1972).

5. Ceres Solver - A Large Scale Non-linear Optimization Library, http://ceres-solver.org, last accessed 2019/02/13. 